\title{
On the Universal Scaling Behavior of the Distance Decay of Plasmon Coupling in Metal Nanoparticle Pairs: A Plasmon Ruler Equation
}

\author{
Prashant K. Jain, ${ }^{\dagger}$ Wenyu Huang, ${ }^{\dagger}$ and Mostafa A. El-Sayed ${ }^{*},,+$, \\ ${ }^{\dagger}$ Laser Dynamics Laboratory, School of Chemistry and Biochemistry, Georgia \\ Institute of Technology, Atlanta, GA 30332-0400, USA. \\ ${ }^{\ddagger}$ Miller Visiting Professor, University of California, Berkeley CA 94720, USA. \\ SUPPORTING INFORMATION
}

* Corresponding author: Mostafa A. El-Sayed: melsayed@gatech.edu, Tel: 404-894-0292, Fax: 404-894-0294. 
DDA Simulations of Au Nanodisc Pairs. The DDA is a discretization procedure for solving the Maxwell's equations, details of which have been described earlier. ${ }^{1,2}$ Briefly, the target structure is geometrically reproduced by a cubic array of $N$ virtual point dipoles with polarizabilities $\alpha_{i}$ located at positions $\boldsymbol{r}_{i}$. In the presence of an incident electric field $\boldsymbol{E}_{o}$, the polarization of each dipole $\boldsymbol{P}_{i}$ is given as:

$$
\boldsymbol{P}_{i}=\alpha_{i} \boldsymbol{E}_{\text {loc }}\left(\boldsymbol{r}_{i}\right)
$$

where $\boldsymbol{E}_{l o c}$ is the local electric field felt by the dipole which is the sum of the incident electric field and the contribution from the other $N-1$ dipoles:

$$
\boldsymbol{E}_{l o c}\left(\boldsymbol{r}_{i}\right)=\boldsymbol{E}_{i n c, i}+\boldsymbol{E}_{o t h e r, i}=\boldsymbol{E}_{o} \exp \left(i \boldsymbol{k} \cdot \boldsymbol{r}_{i}-i \omega t\right)-\sum_{j \neq i} \boldsymbol{A}_{i, j} \cdot \boldsymbol{P}_{j}
$$

where $\boldsymbol{k}$ is the wave vector of the incident plane wave. $\boldsymbol{A}_{i, j}$ is a matrix that represents the interaction between all dipoles as follows:

$$
\boldsymbol{A}_{i, j} \cdot \boldsymbol{P}_{j}=\frac{\exp \left(i k \boldsymbol{r}_{i j}\right)}{\boldsymbol{r}_{i j}{ }^{3}}\left\{k^{2} \boldsymbol{r}_{i j} \times\left(\boldsymbol{r}_{i j} \times \boldsymbol{P}_{j}\right)+\frac{\left(1-i k \boldsymbol{r}_{i j}\right)}{\boldsymbol{r}_{i j}{ }^{2}} \times\left[\boldsymbol{r}_{i j}{ }^{2} \boldsymbol{P}_{j}-3 \boldsymbol{r}_{i j}\left(\boldsymbol{r}_{i j} \cdot \boldsymbol{P}_{j}\right)\right]\right\} \text { for } \mathrm{j} \neq \mathrm{i}
$$

where $\boldsymbol{r}_{i j}=\boldsymbol{r}_{i}-\boldsymbol{r}_{j}$. Starting with an initial guess for the polarizations $\boldsymbol{P}_{j}$, the system of equations represented by eq 2 is solved iteratively for $\boldsymbol{P}_{j}$. The extinction, absorption and scattering crosssection can be then calculated as follows:

$$
\begin{gathered}
C_{e x t}=\frac{4 \pi k}{\left|\boldsymbol{E}_{o}\right|^{2}} \sum_{j=1}^{N} \operatorname{Im}\left(\boldsymbol{E}_{i n c, j}^{*} \cdot \boldsymbol{P}_{j}\right) \\
C_{a b s}=\frac{4 \pi k}{\left|\boldsymbol{E}_{o}\right|^{2}} \sum_{j=1}^{N}\left\{\operatorname{Im}\left[\boldsymbol{P}_{j}\left(\alpha_{j}^{-1}\right)^{*} \boldsymbol{P}_{j}^{*}\right]-\frac{2}{3} k^{3}\left|\boldsymbol{P}_{j}\right|^{2}\right\} \\
C_{s c a}=C_{e x t}-C_{a b s}
\end{gathered}
$$

where Im implies the imaginary part. The extinction, absorption, and scattering efficiencies can be obtained from the respective optical cross-sections by dividing by the effective cross-sectional area $\pi r_{\text {eff }}^{2}$ of the nanostructure. Here $r_{\text {eff }}=(3 V / 4 \pi)^{1 / 3}$ represents the radius of a sphere having a volume equal to that of the target structure. In this work, the DDSCAT 6.1 code developed by Draine and Flatau ${ }^{3}$ was employed for the calculation of the extinction efficiency over the visible wavelength range. Extinction efficiency spectra were formed from the data points at discrete wavelengths by cubic interpolation. The target nanostructure was approximated as a pair of cylinders, each represented by a cubic array of point dipoles with an inter-dipole spacing $\sim 2 \mathrm{~nm}$. The diameter of the cylinder $D$ was $86.5 \mathrm{~nm}$ while the height $h$ was $25.5 \mathrm{~nm}$. The edge-to-edge 
separation between the nanodiscs was varied as $2,8,12,18,28$, and $208 \mathrm{~nm}$. Two other disc diameters, $54.0 \mathrm{~nm}$ and $68.0 \mathrm{~nm}$, were also investigated while keeping the aspect ratio $h / D \approx 0.3$ as well as the inter-dipole spacing constant. Dipole polarizabilities were assigned by the lattice dispersion relation (LDR) by Draine and Goodman, which includes a radiative reaction correction term required for finite dipole arrays. ${ }^{4}$ The dielectric properties of the nanostructure were simulated by the bulk dielectric function for $\mathrm{Au},{ }^{5}$ which as per the DDSCAT procedure was scaled by the dielectric constant $\varepsilon_{m}$ of the surrounding environment/medium. Calculations were performed for different values of $\varepsilon_{m}=1.00,1.38$, and 2.25. The plane wave propagation direction was normal to the plane of the cylinder pair. Calculations were performed for two separate polarization directions, i.e. one along the inter-particle axis, and the other perpendicular to it.

Dipolar-Coupling Model. The electric dipole moment $\mu$ of the single particle in an electric field $E$ is given as

$$
\mu=\alpha \varepsilon_{m} E
$$

where $\alpha$ is the Claussius-Missoti dipole polarizability for an isolated metal nanoparticle in the quasistatic approximation, which is proportional to the particle volume $V$ as:

$$
\alpha=\varepsilon^{0}(1+\gamma)\left(\frac{\varepsilon-\varepsilon_{\mathrm{m}}}{\varepsilon+\gamma \varepsilon_{\mathrm{m}}}\right) \mathrm{V}
$$

where $\varepsilon=\varepsilon_{r}+i \varepsilon_{i}$ is the wavelength dependent dielectric function of bulk gold, $\varepsilon_{m}$ is the medium dielectric constant, $\varepsilon^{0}$ is the vacuum permittivity and $\gamma$ is a factor which depends on the nanoparticle shape, e.g. $\gamma=2$ for spheres. In the presence of a neighboring particle, the electric field felt by each particle is the sum of the incident light field $E$ and the near-field of the electric dipole on the neighboring particle, which decays as the cube of the distance $d$ from the particle:

$$
E^{\prime}=E+\frac{\kappa \mu^{\prime}}{4 \pi \varepsilon_{m} \varepsilon_{0} d^{3}}
$$

$\kappa$ is an orientation factor, which depends on the alignment of the two single-particle dipoles. $\kappa=$ 2 for dipoles arranged head-to-tail corresponding to parallel polarization in our experiments, while $\kappa=-1$ for dipoles aligned side-by-side corresponding to perpendicular polarization. ${ }^{6,7}$ The SPR shift in opposite directions for these two polarizations originates from the opposite sign of $\kappa$ for the two cases. Also since $|\kappa|_{\text {parallel }}>|\kappa|_{\text {perp }}$ the shift magnitude is larger for the parallel 
polarization case (Figure S1). From eq 7 and eq 9, the net polarizability $\alpha$ of the two-particle system is given as

$$
\alpha^{\prime}=\frac{\alpha}{\left(1-\frac{\kappa \alpha}{4 \pi \varepsilon_{0} d^{3}}\right)}
$$

Further we use eq 8 and $V=\pi D^{3} / 6 \Lambda$, where $D$ is the particle dimension along the axis of interparticle interaction and $\Lambda$ depends on the other particle dimensions, e.g. $\Lambda=1$ for spheres and $\Lambda$ $=(1 /$ Aspect ratio $)$ for oblate spheroids interacting along the long-axis.

$$
\alpha^{\prime}=\frac{4 \pi \varepsilon_{0} D^{3}\left(\varepsilon-\varepsilon_{m}\right)(1+\gamma)}{\varepsilon\left(24 \Lambda-\frac{\kappa(1+\gamma) D^{3}}{d^{3}}\right)+\varepsilon_{m}\left(24 \Lambda \gamma+\frac{\kappa(1+\gamma) D^{3}}{d^{3}}\right)}
$$

The SPR condition is thus specified by the maximum of the polarizability as:

$$
\left.\varepsilon_{\mathrm{r}}\right|_{\mathrm{SPmax}}=-\varepsilon_{\mathrm{m}}\left(\frac{\gamma(\mathrm{s} / \mathrm{D}+1)^{3}+\kappa(1+\gamma) / 24 \Lambda}{(\mathrm{s} / \mathrm{D}+1)^{3}-\kappa(1+\gamma) / 24 \Lambda}\right)
$$

since $s=d+D$. We can now utilize the near-linear dependence of $\varepsilon_{r}$ on wavelength in the visible (500-700 nm) region for $\mathrm{Au}, \lambda=464.6-14.3 \varepsilon_{\mathrm{r}}$ and get:

$$
\left.\lambda\right|_{S P \max }=464.6+14.3 \varepsilon_{m}\left(\frac{\gamma(s / D+1)^{3}+\kappa(1+\gamma) / 24 \Lambda}{(s / D+1)^{3}-\kappa(1+\gamma) / 24 \Lambda}\right)
$$

Denoting the single-particle resonance wavelength maximum as $\lambda_{0}$ (for $\mathrm{s} / \mathrm{D} \rightarrow \infty$ ) we get for the parallel polarization, i.e. $\kappa=2$ :

$$
\Delta \lambda / \lambda_{0}=\frac{14.3 \varepsilon_{m}(1+\gamma)^{2}}{\left(12 \Lambda(\mathrm{s} / \mathrm{D}+1)^{3}-(1+\gamma)\left(464.6+14.3 \varepsilon_{\mathrm{m}} \gamma\right)\right.}
$$

From eq 14 , we see that $\Delta \lambda / \lambda_{0}$ is a rational function of $s / D$, which can be approximated very nearly to an exponential decay (Figure S1). This function has a somewhat smoother curvature than the exponential function - a behavior, which is also reflected in the exponential fits to our experimental and DDA simulation plots. While the function predicted by the dipolar model explains the near-exponential decay, the estimated $\tau$ value of 0.40 is much higher than that observed in the experiments and DDA simulations, suggesting that the dipolar model underestimates the coupling strength at smaller inter-particle gaps. ${ }^{8,9}$ Nevertheless, the dipolar model can be used for qualitative elucidation of the decay of the plasmon coupling. 
The part of the rational function in eq 14: $f(s / D)=\frac{1}{\left(12 \Lambda(s / D+1)^{3}-(1+\gamma)\right)}$, which governs the decay rate of $\Delta \lambda / \lambda_{0}$ with respect to $s / D$, has no functional dependence on the medium dielectric constant or the metal type. The effect of shape on the decay rate is also weak as shown in Figure S2. The other parameters in the function only influence the amplitude of the decay and this includes the effect of the medium dielectric constant $\left(\varepsilon_{m}\right)$, the metal type (through the relationship between $\lambda$ and $\varepsilon_{r}$ ) and the nanoparticle shape (through $\Lambda$ and $\gamma$ ). As an example, the dependence of the amplitude on $\varepsilon_{m}$ is shown in Figure S3. 


\section{SUPPORTING FIGURES}

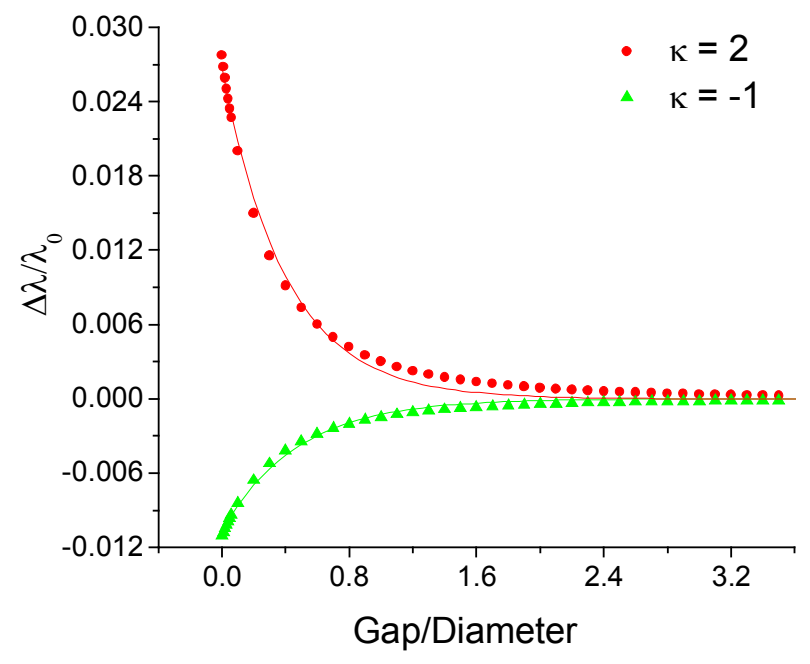

Figure S1. Plot of the fractional shift as a function of gap/diameter calculated using the dipolar-coupling model (eq 14) for our Au nanodisc pair system, modeled by a pair of oblate spheroids of aspect ratio 0.28 , interacting along their long axis for parallel polarization, i.e. $\kappa=2$, and perpendicular polarization, i.e. $\kappa=-1$, in a medium of dielectric constant $=1.0$. Solid curves are fits to the single exponential decay $y=a . \mathrm{e}^{-\mathrm{x} / \tau}$ where $\tau=0.40 \pm 0.01, a=$ 0.03 for parallel polarization whereas $\tau=0.47 \pm 0.01, a=-0.01$ for perpendicular polarization. 


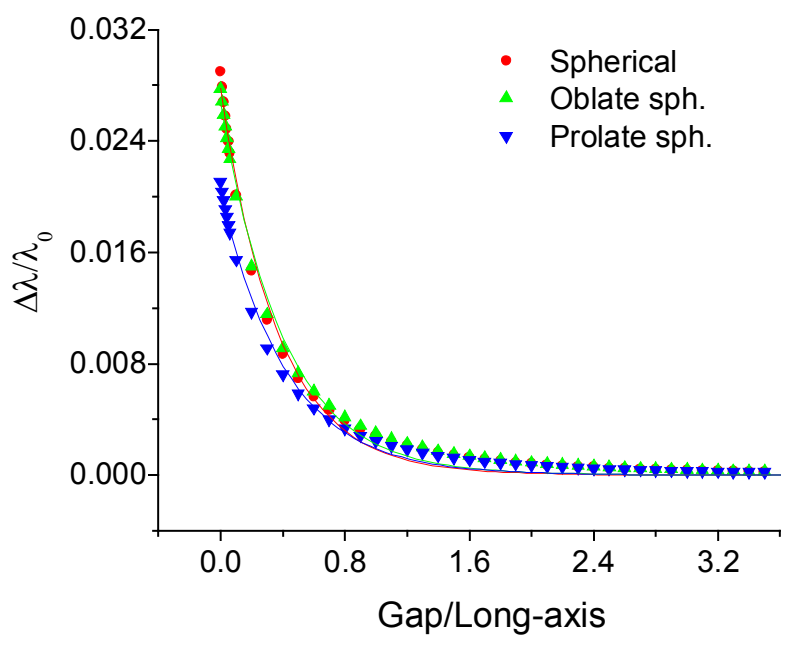

Figure S2. Plot of the fractional shift versus the ratio of gap to long-axis dimension for Au nanoparticle pairs for different shapes: spherical, oblate spheroids of aspect ratio 0.28 and prolate spheroids of aspect ratio 3.0, calculated using the dipolar-coupling model (eq 14) for parallel polarization and medium dielectric constant $=1.0$. The interaction of the spheroids was considered along their long axis. The solid curves represent least-square fits to the exponential decay $y=a . \mathrm{e}^{-\mathrm{x} / \tau}$ where $\tau=0.37 \pm 0.01,0.40 \pm 0.01$ and $0.42 \pm 0.01$ and amplitude $a=0.028,0.027$ and 0.020 for spherical, oblate and prolate shapes respectively. 


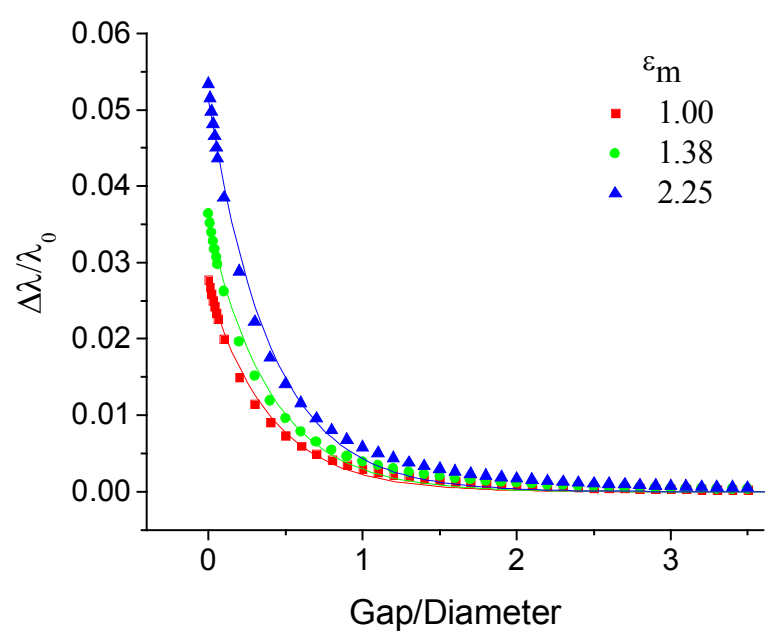

Figure S3. Plot of the fractional shift versus gap/diameter for Au nanodisc pair under parallel polarization for different medium dielectric constants, calculated using the dipolar-coupling model (eq 14). The solid curves are least-squares fits to the single exponential decay $y=a \cdot \mathrm{e}^{-\mathrm{x} / \tau}$ showing similar decay constant $\tau=0.40$ for the different medium dielectric constants but increasing amplitude $a=0.027,0.035$ and 0.051 for $\varepsilon_{m}=1.00,1.38$ and 2.25 respectively. 


\section{SUPPORTING REFERENCES:}

(1) Draine, B. T.; Flatau, P. J. J. Opt. Soc. Am. A 1994, 11, 1491-1499.

(2) Lee, K.-S.; El-Sayed, M. A. J. Phys. Chem. B 2005, 109, 20331-20338.

(3) Draine, B. T.; Flatau, P. J. http://arxiv.org/abs/astro-ph/0409262v2 2004.

(4) Draine, B. T.; Goodman, J. Astrophysical Journal 1993, 405, 685-697.

(5) Johnson, P. B.; Christy, R. W. Phys. Rev. B 1972, 6, 4370-4379.

(6) Jain, P. K.; Eustis, S.; El-Sayed, M. A. J. Phys. Chem. B 2006, 110, 18243-18253.

(7) Gluodenis, M.; Foss, C. A., Jr. J. Phys. Chem. B 2002, 106, 9484-9489.

(8) Gunnarsson, L.; Rindzevicius, T.; Prikulis, J.; Kasemo, B.; Käll, M.; Zou, S.; Schatz, G. C. J. Phys. Chem. B 2005, 109, 1079 -1087.

(9) Nordlander, P.; Oubre, C.; Prodan, E.; Li, K.; Stockman, M. I. Nano Lett. 2004, 4, 899-903. 